\title{
Correlation potential and ladder diagrams
}

\author{
V. A. Dzuba \\ School of Physics, University of New South Wales, Sydney 2052, Australia
}

(Dated: November 20, 2018)

\begin{abstract}
The all-order correlation potential method of accurate atomic structure calculations for atoms with one external electron is extended to include one more class of correlation diagrams to all orders. These are the so-called ladder diagrams which describe residual Coulomb interaction between an external electron and atomic core. This is in addition to the screening of Coulomb interaction by core electrons and the hole-particle interaction in the core polarization operator which are also included in all orders. Calculations of the energies of the lowest $s, p$ and $d$ states of cesium and thallium show that inclusion of the ladder diagrams leads to significant improvement of the accuracy of the calculations. The discrepancy between theoretical and experimental energies is reduced to a small fraction of a per cent in all cases. This widens the range of atoms and atomic states for which the correlation potential method can produce very accurate results.
\end{abstract}

PACS numbers: 31.15.A-, 31.15.V-

\section{INTRODUCTION}

There are many areas in modern physics which require accurate atomic calculations. This includes parity and time invariance violation in atoms [1, 2], search for variation of the fundamental constants [3, 4, 5], atomic clocks [6, 7], etc. Calculations are needed for planing of the experiments and for interpretation of the results. Accuracy of atomic calculations is often a limitation factor. For example, the most accurate measurements of the parity non-conservation (PNC) in atoms was done in Boulder in 1997 for the cesium atom [8]. The accuracy of the measurements is $0.35 \%$. Theoretical accuracy of best calculations is on the level of $0.4-0.5 \%$ and as a result the accuracy of extraction of the weak charge of the cesium nucleus is only $0.6 \%$ 2]. The situation is even worse for the PNC in thallium where best experimental accuracy is $1 \%$ [9] while the accuracy of best calculations is $3 \%$ [10] and 2.5\% [11]. Since atomic PNC measurements serve as an important source of information about low energy physics and possible extensions to the standard model, further improvements in the accuracy of atomic calculations is highly desirable.

In present paper we limit our discussion to monovalent atoms keeping in mind $\mathrm{PNC}$ in $\mathrm{Cs}$ and $\mathrm{Tl}$ and other similar important applications. Ground state configuration of thallium is [Xe] $5 d^{10} 6 s^{2} 6 p$ and to some extend it can be treated as an atom with one external electron above the $[\mathrm{Xe}] 5 d^{10} 6 s^{2}$ closed-shell core. This approach was used in our early calculations of the PNC in Tl [10]. It is generally believed however that for more accurate results $\mathrm{Tl}$ should be treated as an atom with three external electrons above the $[\mathrm{Xe}] 5 d^{10}$ closed-shell core. This is because the $6 s$ electrons are easy to excite which is evident from the existence of the states in thallium discrete spectrum which belong to the $6 s 6 p^{2}$ configuration. A method which combines the configuration interaction (CI) technique for valence electrons with the many-body perturbation theory (MBPT) for the core-valence correlations was suggested in Ref. [12]. Recent calculations of the Tl PNC performed with the use of this method [1] achieved only moderate improvement of accuracy, from $3 \%$ in Ref. [10] to $2.5 \%$ in Ref. [11].

In present paper we advocate a different approach which treats thallium atom as a monovalent system but includes dominating classes of core-valence correlations in all orders. It is based on the all-order correlation potential method which was developed in Ref. [13] and used in a number of calculations mostly for alkali-metal atoms [14, 15, 16, 17, 18]. To make it work equally well for other atoms like thallium we extend the technique to include one more class of higher-order diagrams, the ladder diagrams. This diagrams describe residual Coulomb interaction of the external electron with the core. The idea of the extension is inspired by the coupled-cluster (CC) approach. This is another powerful method which is widely used for monovalent atoms. In this approach, the many-electron wave function of an atoms is written in terms of single, double, etc. excitations from the reference Hartree-Fock wave function. The accuracy depends on the number of terms included into expansion and limited by available computer power. The method was used by many groups for accurate calculations of wide range of properties of many-electrons atoms (see, e.g. Ref. [19]) including PNC in Cs [20]. There are plans to use this approach to improve the accuracy of calculations of the PNC in Cs by including more terms into the expansion [21].

Although the CC approach can produce very accurate results it is computationally very demanding. Even relatively simple singe-double approximation takes significant computer resources but insufficiently accurate for some atoms, e.g. heavy alkali-metal atoms [22]. In contrast, the all-order correlation potential method is very efficient. However, it produces accurate results only for $s$ and $p$ states of alkali-metal atoms. The accuracy for other monovalent atoms and for the $d$ states of alkalimetal atoms is significantly lower. In general, the overlap between wave functions of the valence and core electrons must be small for accurate results. Small overlap 

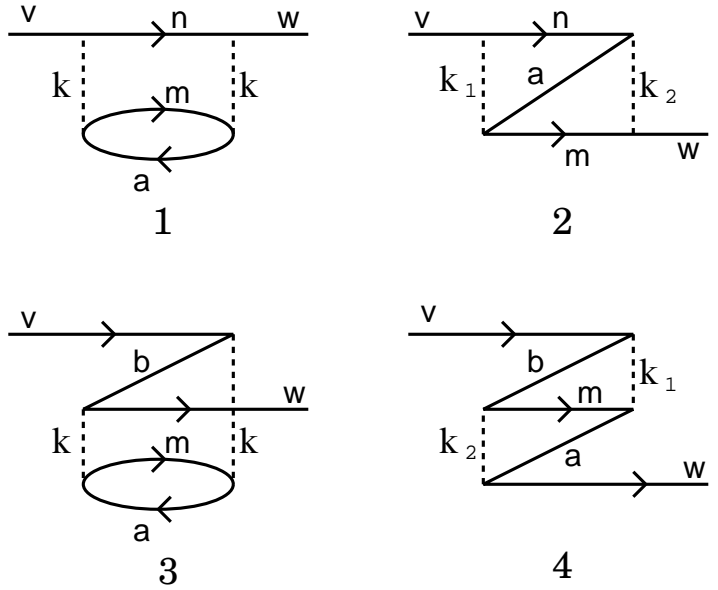

FIG. 1: Second order correlation diagrams for $\hat{\Sigma}$

would mean small residual Coulomb interaction between valence and core electrons. To overcome this limitation of the all-order correlation potential method we use the CClike equations to include residual Coulomb interaction between valence and core electrons in all orders. Corresponding terms in the $\mathrm{MBPT}$ are presented by ladder diagrams.

To test the technique we calculate lowest $s, p$ and $d$ energy levels of cesium and thallium. Consideration of the lowest states is sufficient for testing of the calculations of the correlations. This is because correlations are smaller for excited states and within the same technique accuracy of calculations is usually better for excited states. Therefore, wide range of the different states of monovalent atoms are covered.

We demonstrate that the inclusion of the ladder diagrams leads to significant improvements in the accuracy of calculation. This opens a way of atomic structure calculations for many important applications with the accuracy which was not available before.

\section{CORRELATION POTENTIAL}

The all-order correlation potential method was developed in Refs. 13 and successfully used for a number of calculations for alkali-metal atoms and their isoelectronic ions. The method is based on the use of the so-called correlation potential $\hat{\Sigma}$ which is defined in such a way that its expectation value over a wave function $|v\rangle$ of a valence electron is equal to the many-body perturbation theory (MBPT) expression for the correlation correction to the energy of the electron

$$
\delta \epsilon_{v}=\langle v|\hat{\Sigma}| v\rangle
$$

The correlation potential $\hat{\Sigma} \equiv \Sigma_{v}\left(r_{1}, r_{2}\right)$ is a non-local operator similar to the Hartree-Fock (HF) exchange potential. It can be used in the HF equations for valence electrons to calculate the so-called Brueckner orbitals

$$
\left(\hat{H}^{H F}+\hat{\Sigma}-\epsilon_{v}\right) \psi_{v}=0
$$

Here $\hat{H}^{H F}$ is the HF Hamiltonian. Solving the equation (2) for different states of external electron produces the wave functions and the energies which include correlations.

Following our earlier works [13, 14, 15, 16, 17] we use the all-order correlation potential $\hat{\Sigma}^{(\infty)}$ which includes two classes of higher-order correlations: (a) screening of Coulomb interaction between a valence electron and a core electron by other core electrons and (b) an interaction between an electron excited from the core and a hole created by this excitation. One more class of higherorder diagrams, iteration of the $\hat{\Sigma}$-operator, is included by iterating the equations for Brueckner orbitals (2).

The MBPT expansion for the correlation correction operator $\hat{\Sigma}$ starts from the second order. All four Brueckner-Goldstone diagrams are shown on Fig. 1 (to be more precise matrix elements $\left\langle v\left|\hat{\Sigma}^{(2)}\right| w\right\rangle$ of the secondorder correlation potential $\hat{\Sigma}^{(2)}$ are shown). However, it is more convenient to use the Feynman diagram technique to include dominating higher-order correlations. We do this for direct diagrams 1 and 3 on Fig. 1. Direct diagrams strongly dominate over exchange ones in most of the cases and require accurate treatment. The higher-order effects for exchange diagrams (2 and 4 on Fig. 11) are included in a semi-empirical way by introducing screening factors as it will be explained below.

Screening of Coulomb interaction are included by inserting core polarization loops into Coulomb lines as shown on Fig. 2. Hole-particle interaction in the polarization operator is shown on Fig. 3. The all-order $\hat{\Sigma}$ operator is shown on Fig. 4. This operator is drawn using Feynman diagram technique, screened Coulomb interaction (Fig. 2) and the core-polarization operator with the hole-particle interaction in it (Fig. 3). This diagram does not include exchange terms. Exchange diagrams are much smaller and screening for them is included in a semi-empirical way via screening factors $f_{k}$. It is assumed that screening depends only on the multipolarity of the Coulomb interaction $k$ and every Coulomb integral $g_{k}$ in diagrams 2 and 4 on Fig. 1 is replaced by $f_{k} g_{k}$, where screening factors $f_{k}$ are found from the calculation of the direct diagram (Fig. 4). The values of the screening factors for $\mathrm{Cs}$ and $\mathrm{Tl}$ are given in Table $\mathrm{I}$.

The results of the calculations for $\mathrm{Ca}$ and $\mathrm{Tl}$ with the all-order correlation potential method are presented in Table II. The final results are very accurate for the $s$ and $p$ states of Cs and $s$ and $d$ states of Tl. In general, the all-order correlation potential method gives very accurate results for systems in which external electron is on large distances from the atomic core and its residual Coulomb interaction with the core is small. This is because this interaction is included in the second-order of the MBPT only. This remains true even when all three classes of the higher-order diagrams discussed above are 


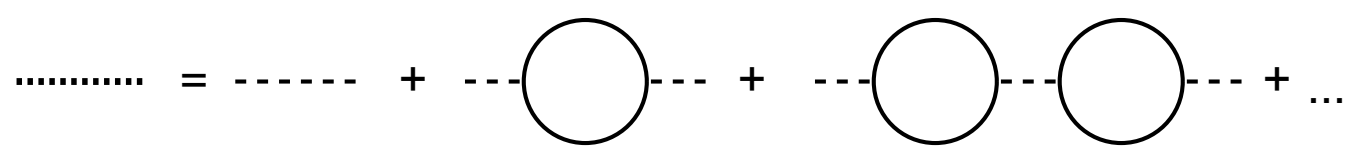

FIG. 2: Screening of Coulomb interaction by polarization of the atomic core

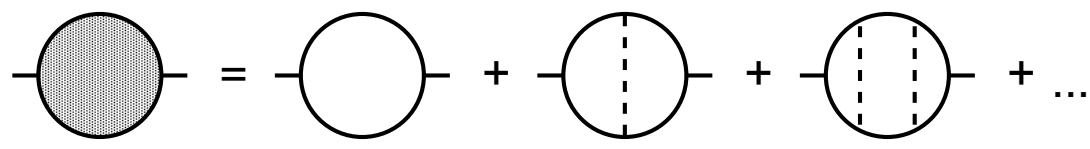

FIG. 3: Hole-particle interaction in the polarization operator

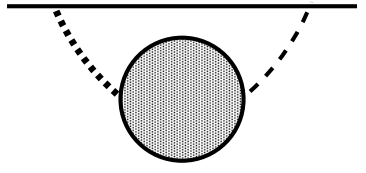

FIG. 4: All-order correlation potential $\hat{\Sigma}$

TABLE I: Screening factors $f_{k}$ to calculate exchange diagrams of $\hat{\Sigma}$.

\begin{tabular}{cllllllll}
\hline \hline Atom & State & $f_{0}$ & $f_{1}$ & $f_{2}$ & $f_{3}$ & $f_{4}$ & $f_{5}$ & $f_{6}$ \\
\hline Cs & All & 0.72 & 0.62 & 0.83 & 0.89 & 0.94 & 1.00 & 1.00 \\
Tl & $7 s_{1 / 2}$ & 0.54 & 0.55 & 0.90 & 0.89 & 0.95 & 0.97 & 0.99 \\
& $6 p_{1 / 2}$ & 0.71 & 0.67 & 0.85 & 0.90 & 0.95 & 0.97 & 0.99 \\
& $6 p_{3 / 2}$ & 0.74 & 0.58 & 0.86 & 0.89 & 0.97 & 0.97 & 0.99 \\
& $6 d_{3 / 2}$ & -.19 & 0.54 & 0.90 & 0.92 & 0.97 & 0.98 & 0.99 \\
& $6 d_{5 / 2}$ & 0.14 & 0.55 & 0.90 & 0.92 & 0.97 & 0.98 & 0.99 \\
\hline \hline
\end{tabular}

included. Second order is insufficient for cases of large overlap between wave functions of the core and the valence electron. This is the case for the ground state of $\mathrm{Tl}$ due to large overlap between the $6 s$ and $6 p$ states as well as for the $5 d$ states of Cs due to large overlap between

TABLE II: Energies of the lowest $s, p$ and $d$ states of Cs and $\mathrm{Tl}$ in different approximations $\left(\mathrm{cm}^{-1}\right)$; comparison with experiment.

\begin{tabular}{crrrrrr}
\hline \hline Atom & State & RHF & $\Sigma^{(2) a}$ & $\Sigma^{(\infty) b}$ & $\Delta^{c}$ & Exp.[23] \\
\hline Cs & $6 s_{1 / 2}$ & 27954 & 32377 & 31462 & -55 & 31407 \\
& $6 p_{1 / 2}$ & 18791 & 20523 & 20296 & -67 & 20229 \\
& $6 p_{3 / 2}$ & 18389 & 19927 & 19728 & -53 & 19675 \\
& $5 d_{3 / 2}$ & 14138 & 17459 & 17166 & -258 & 16908 \\
& $5 d_{5 / 2}$ & 14163 & 17305 & 17050 & -240 & 16810 \\
$\mathrm{Tl}$ & $7 s_{1 / 2}$ & 21109 & 23375 & 22887 & -101 & 22786 \\
& $6 p_{1 / 2}$ & 43823 & 51597 & 50815 & -1551 & 49264 \\
& $6 p_{3 / 2}$ & 36636 & 43524 & 42491 & -1020 & 41471 \\
& $6 d_{3 / 2}$ & 12217 & 13428 & 13296 & -150 & 13146 \\
& $6 d_{5 / 2}$ & 12167 & 13319 & 13160 & -96 & 13064 \\
\hline \hline
\end{tabular}

${ }^{a}$ Brueckner orbitals with the second-order $\hat{\Sigma}$

${ }^{b}$ Brueckner orbitals with the all-order $\hat{\Sigma}$

${ }^{c} \Delta=\mathrm{E}_{\exp }-\mathrm{E}_{\text {calc }}\left(\hat{\Sigma}^{(\infty)}\right.$ the $5 d$ and $5 p$ states. Here one needs to include residual Coulomb interaction between external electron and atomic core in all-orders to get accurate results. This can be done via the so called ladder diagrams which will be discussed in next section.

In the end of this section we present on Fig. 15 all third order diagrams corresponding to the the all-order correlation potential method. The purpose is to demonstrate that there is no overlap between the higher-order diagrams of the all-order correlation potential method and the ladder diagrams of the next section. No overlap means no double counting.

\section{LADDER DIAGRAMS}

As it has been discussed in previous section, in atoms with large overlap between the wave wave functions of the core and valence electrons the residual Coulomb interaction between external electron and the core should be included in all orders. This can be done via the so called ladder diagrams. An example of the third and forth order ladder diagrams are shown on Fig. 6. In higher orders such diagrams have many parallel Coulomb lines representing interaction of the external electrons with the core. This makes a diagram to look like a ladder. Here is the name.

Direct calculation of the higher-order ladder diagrams is impractical. A much more efficient way is to perform an appropriate iteration procedure in which each iteration corresponds to next order of the MBPT but takes exactly the same time. For example, diagrams on Fig. 6 can be obtained by iteration the graphical equation of Fig. (7. Here solid line represents an effective Coulomb interaction which is initially equal to the ordinary Coulomb interaction and then adds a Coulomb line to itself with every new iteration. Figs. 6 and 7 do not represent all ladder diagrams. They only show some higher-order extensions of the diagram 1 of Fig. 1. To include all ladder diagrams we must make sure that all Coulomb lines in all four diagrams of Fig. 1 are repeated many times in the cause of iterations. Extra Coulomb lines must be connected to the lines of electrons excited from the core as well as to the lines of the holes created in the core by 


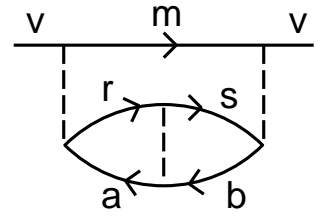

a1

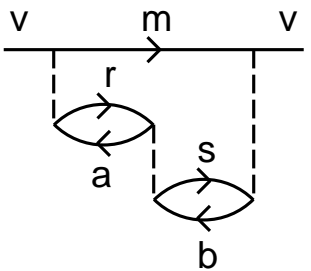

a3

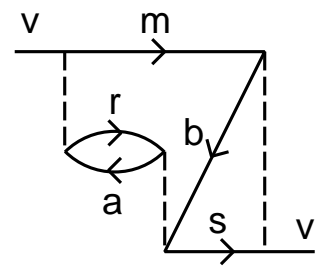

a7

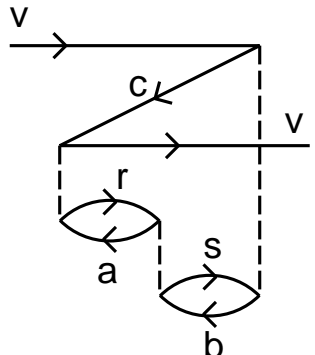

a4

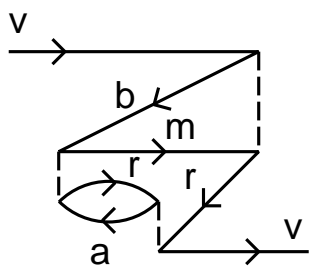

a8

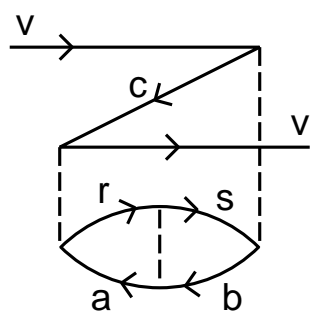

a2

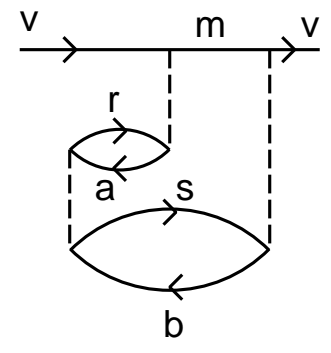

a5

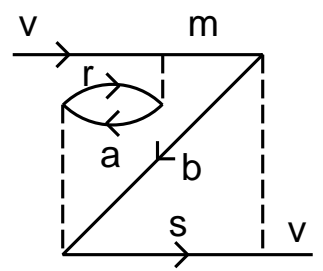

a9

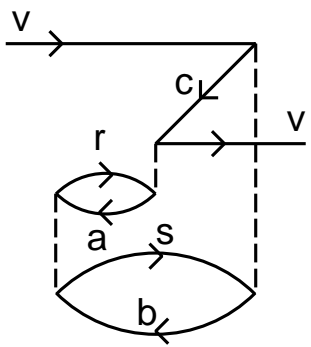

a6

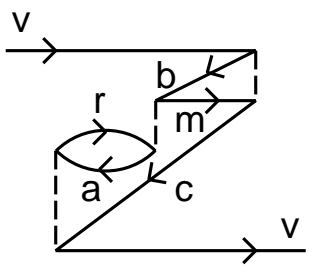

a10

FIG. 5: Third order diagrams corresponding to the correlation potential method. Diagrams a1 and a2 include hole-particle interaction. Other diagrams include screening of the Coulomb interaction. Diagrams a5-a10 have mirror-reflection partners. Exchange diagrams are not shown.
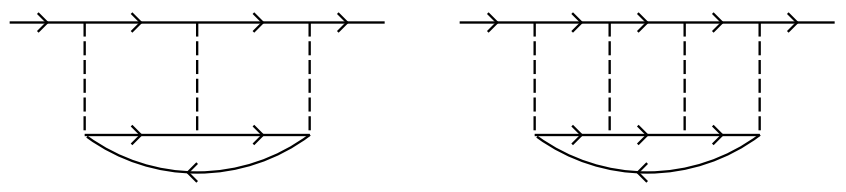

FIG. 6: Sample third and forth order ladder diagrams.

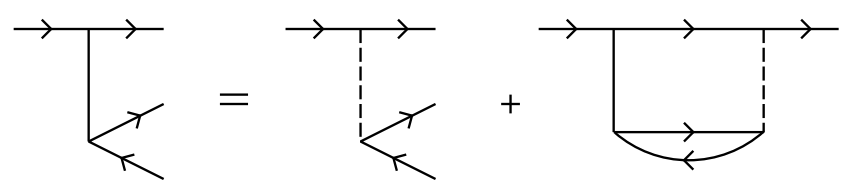

FIG. 7: Graphic equation which generates ladder diagrams on Fig. 6

electron excitations. For example, apart from diagrams on Fig. 6 there are must be companion diagrams in which arrows in the lower loop go opposite direction.

The equations which satisfy these conditions can be written as two sets of equations. The first is for atomic core:

$$
\begin{aligned}
& \left(\epsilon_{a}+\epsilon_{b}-\epsilon_{m}-\epsilon_{n}\right) \rho_{m n a b}=g_{m n a b}+ \\
& \sum_{r s} g_{m n r s} \rho_{r s a b}+\sum_{r c}\left(g_{c n b r} \rho_{m r c a}+g_{c m a r} \rho_{n r c b}\right) .
\end{aligned}
$$

And another is for a specific state $v$ of an external electron:

$$
\begin{aligned}
& \left(\epsilon_{v}+\epsilon_{b}-\epsilon_{m}-\epsilon_{n}\right) \rho_{m n v b}=g_{m n v b}+ \\
& \sum_{r s} g_{m n r s} \rho_{r s v b}+\sum_{r c}\left(g_{c n b r} \rho_{m r c v}+g_{c m v r} \rho_{n r c b}\right) .
\end{aligned}
$$

Here parameters $g$ are Coulomb integrals

$$
g_{m n a b}=\iint \psi_{m}^{\dagger}\left(r_{1}\right) \psi_{n}^{\dagger}\left(r_{2}\right) e^{2} / r_{12} \psi_{a}\left(r_{1}\right) \psi_{b}\left(r_{2}\right) d \mathbf{r}_{1} d \mathbf{r}_{2},
$$

variables $\rho$ are the coefficients representing expansion of the atomic wave function over double excitations from the zero-order Hartree-Fock reference wave function; parameters $\epsilon$ are the single-electron Hartree-Fock energies. Coefficients $\rho$ are to be found by solving the equations 
iteratively starting from

$$
\rho_{m n i j}=\frac{g_{m n i j}}{\epsilon_{i}+\epsilon_{j}-\epsilon_{m}-\epsilon_{n}} .
$$

Indexes $a, b, c$ numerate states in atomic core, indexes $m, n, r, s$ numerate states above the core, indexes $i, j$ numerate any states.

The equations for the core (3) do not depend on the valence state $v$ and are iterated first. The convergence is controlled by the correction to the core energy

$$
\delta E_{C}=\frac{1}{2} \sum_{a b m n} g_{a b m n} \tilde{\rho}_{m n a b}
$$

where

$$
\tilde{\rho}_{m n a b}=\rho_{m n a b}-\rho_{m n b a} .
$$

When iterations for the core are finished the equations (4) are iterated for as many valence states $v$ as needed.

Correction to the energy of the valence state $v$ arising from the iterations of equations (3) and (4) is given by

$$
\delta \epsilon_{v}=\sum_{m a b} g_{a b v m} \tilde{\rho}_{m v a b}+\sum_{m n b} g_{v b m n} \tilde{\rho}_{m n v b} .
$$

The equations (3) and (4) are very similar to the well-known linearized coupled-cluster single-double (SD) equations (see, e.g. [24]). However, certain terms are removed from the SD equation to arrive to Eqs. (3) and (4). This is because we are going to combine the equations with the correlation potential method and removal of the terms is needed to ensure no double counting. Only terms corresponding to the ladder diagrams need to be included.

Since Brueckner energy $\epsilon_{v}$, in the equation (2) and the correction $\delta \epsilon_{v}$, in the equation (6) both include the second-order correlation correction, it is convenient to define the correction associated with the ladder diagrams as a difference

$$
\delta \epsilon_{v}^{(l)}=\delta \epsilon_{v}-\left\langle v\left|\hat{\Sigma}^{(2)}\right| v\right\rangle .
$$

Here $\hat{\Sigma}^{(2)}$ is the second-order correlation potential given by four diagrams on Fig. 1]. The correction (7) is additional to the corrections considered in previous section.

If equations (3) and (4) are iterated simultaneously, i.e. one iteration is done for Eq. (3) and one iteration is done for Eq. (4), and then process is repeated again, etc., then every iteration corresponds to the next order of the MBPT. For example, single iteration of both sets of equations produces the third-order ladder diagrams. All third-order ladder diagrams are shown on Fig. 8. Comparison with the third order correlation potential diagrams presented on Fig. 5 shows that there is no overlap between them. This means that ladder diagrams represent a new class of the higher-order diagrams which was not included into the correlation potential method.

\begin{tabular}{|c|c|c|c|}
\hline \multicolumn{2}{|c|}{ Cs } & \multicolumn{2}{|c|}{$\mathrm{Tl}$} \\
\hline State & $\delta \epsilon_{v}^{(l)}$ & State & $\delta \epsilon_{v}^{(l)}$ \\
\hline $6 s_{1 / 2}$ & -131 & $7 s_{1 / 2}$ & -43 \\
\hline $6 p_{1 / 2}$ & -60 & $6 p_{1 / 2}$ & -1215 \\
\hline $6 p_{3 / 2}$ & -54 & $6 p_{3 / 2}$ & -794 \\
\hline $5 d_{3 / 2}$ & -189 & $6 d_{3 / 2}$ & -29 \\
\hline $5 d_{5 / 2}$ & -193 & $6 d_{5 / 2}$ & -27 \\
\hline
\end{tabular}

The results of the calculations of the ladder correction $\delta \epsilon_{v}^{(l)}$ for the lowest $s, p$ and $d$ states of Cs and Tl are
TABLE III: Ladder diagrams corrections to the energies of the lowest $s, p$ and $d$ states of $\mathrm{Cs}$ and $\mathrm{Tl}\left(\mathrm{cm}^{-1}\right)$.

presented in Table III Comparison with the data from Table II shows that inclusion of the ladder diagrams leads to significant improvements of the accuracy of the results in practically all cases. However, these results are not final since we are going to include ladder diagrams into the correlation potential $\hat{\Sigma}$. This would slightly change the results. Apart from that, small difference between theory and experiment after inclusion of the ladder diagrams means that other small corrections such as Breit interaction and quantum electrodynamic corrections (QED) need to be considered as well. This will be done in next section.

\section{CALCULATIONS FOR CESIUM AND THALLIUM}

It has been demonstrated in previous section that the calculations of the energy levels of such atoms as cesium and thallium by means of the all-order correlation potential method can be significantly improved if contributions of the ladder diagrams are also included. The remaining discrepancy between theory and experiment is a fraction of a per cent for all low $s, p$ and $d$ states of both atoms. This means that other small corrections to the energy need to be considered.

In this section we consider inclusion of the ladder diagrams into the correlation potential $\hat{\Sigma}$ as well as the Breit and QED corrections.

\section{A. Inclusion of the ladder diagrams into the correlation potential}

For accurate calculations of the matrix elements its is important to have as accurate correlation potential $\hat{\Sigma}$ as possible. This would allow to have accurate Brueckner orbitals by solving the equations (2) for valence electrons. It would also lead to more accurate values of such corrections to the matrix elements as structure radiation and renormalization which can also be expressed in terms of $\hat{\Sigma}$ [25]. Therefore, it is important to include ladder diagrams into the correlation potential $\hat{\Sigma}$. This is done by modifying the expressions for the second-order correlation potential $\hat{\Sigma}^{(2)}$ (Fig. 1). Each term for the diagrams 


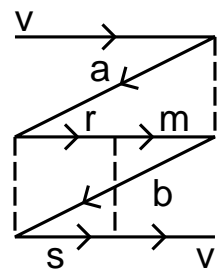

L1

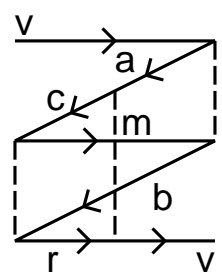

L5

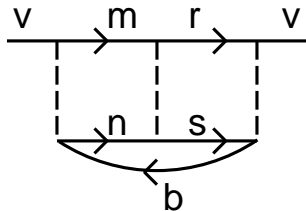

L9

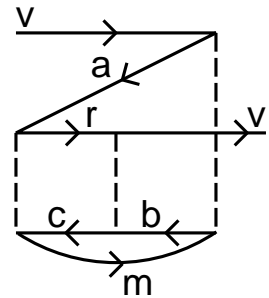

L2

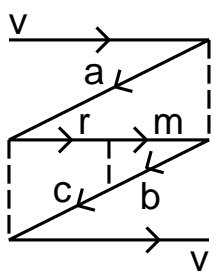

L6

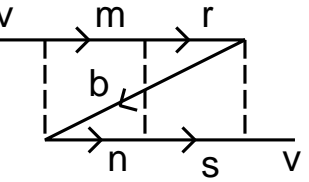

L10

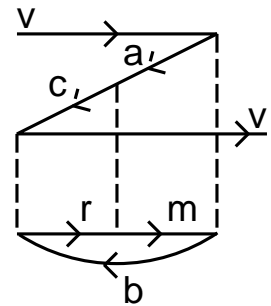

L3

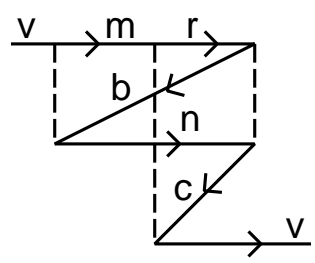

L7

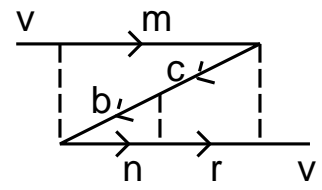

L11

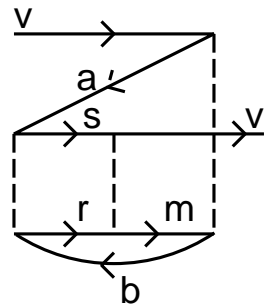

L4

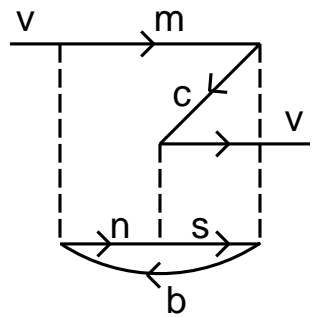

L8

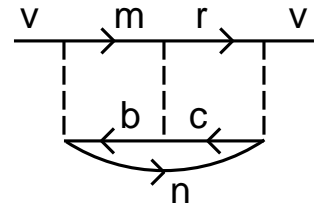

L12

FIG. 8: Third order ladder diagrams.

1 and 2 on Fig. 1 is multiplied by the factor

$$
\frac{\rho_{\text {vanm }}}{g_{\text {vanm }}\left(\epsilon_{v}+\epsilon_{a}-\epsilon_{n}-\epsilon_{m}\right)}-1,
$$

and each term for the diagrams 3 and 4 is multiplied by a similar factor

$$
\frac{\rho_{v a b m}}{g_{v a b m}\left(\epsilon_{a}+\epsilon_{b}-\epsilon_{v}-\epsilon_{m}\right)}-1,
$$

The meaning of $\rho, g, \epsilon$ and all indexes is the same as in equation (3) and (4). Subtraction of one is needed to exclude double counting of the second-order correlation potential $\hat{\Sigma}^{(2)}$ (as in (77)).

The results of the calculations of the energy levels of Cs and $\mathrm{Tl}$ with the all-order $\hat{\Sigma}$ which also includes ladder diagrams presented in Table IV together with the Breit and QED corrections.

\section{B. Breit and QED corrections}

The Breit interaction accounts for magnetic and retardation corrections to the non-relativistic Coulomb interaction between atomic electrons. We use the following form for the Breit operator,

$$
\hat{H}^{B}=-\frac{\boldsymbol{\alpha}_{1} \cdot \boldsymbol{\alpha}_{2}+\left(\boldsymbol{\alpha}_{1} \cdot \mathbf{n}\right)\left(\boldsymbol{\alpha}_{2} \cdot \mathbf{n}\right)}{2 r},
$$

where $\mathbf{r}=\mathbf{n} r, r$ is the distance between electrons, and $\boldsymbol{\alpha}$ is the Dirac matrix.

In a similar way to the Coulomb interaction, we determine the self-consistent Hartree-Fock contribution arising from Breit. This is found by solving the Hartree-Fock equations for single-electron orbitals in the potential

$$
\hat{V}=V^{C}+V^{B},
$$

where $V^{C}$ is the Coulomb potential, $V^{B}$ is the Breit potential. Coulomb interaction in the second-order $\hat{\Sigma}$ (Fig. 1) is also modified to include Breit operator (8). The Breit correction to the energy of external electron is found by comparing the second-order Brueckner energies (Eq. (2)) calculated with and without Breit interaction.

Quantum electrodynamics radiative corrections to the energies (Lamb shifts) are accounted for by use of the radiative potential introduced in Ref. 26]. This potential has the form

$$
V_{\mathrm{rad}}(r)=V_{U}(r)+V_{g}(r)+V_{e}(r),
$$

where $V_{U}$ is the Uehling potential, $V_{g}$ is the potential arising from the magnetic formfactor, and $V_{e}$ is the potential arising from the electric formfactor. As for the case of Breit interaction, the QED corrections to the energies of external electron are found by solving equations (2) with and without radiative potential. 
TABLE IV: Final results for the energies of the lowest $s, p$ and $d$ states of $\mathrm{Cs}$ and $\mathrm{Tl}\left(\mathrm{cm}^{-1}\right)$; comparison with experiment.

\begin{tabular}{cccrrrrr}
\hline \hline Atom & State & $\hat{\Sigma}^{a}$ & Breit & QED & Sum & $\Delta^{b}$ & Exp. [23] \\
\hline Cs & $6 s_{1 / 2}$ & 31402 & -4 & -22 & 31376 & 31 & 31407 \\
& $6 p_{1 / 2}$ & 20191 & -10 & 1 & 20182 & 47 & 20229 \\
& $6 p_{3 / 2}$ & 19632 & -4 & 0 & 19628 & 47 & 19675 \\
& $5 d_{3 / 2}$ & 16901 & 20 & 5 & 16926 & -18 & 16908 \\
& $5 d_{5 / 2}$ & 16814 & 21 & 4 & 16839 & -29 & 16810 \\
$\mathrm{Tl}$ & $7 s_{1 / 2}$ & 22943 & -26 & -24 & 22893 & -107 & 22786 \\
& $6 p_{1 / 2}$ & 49466 & -251 & 38 & 49253 & 11 & 49264 \\
& $6 p_{3 / 2}$ & 41613 & -126 & 29 & 41516 & -45 & 41471 \\
& $6 d_{3 / 2}$ & 13239 & -7 & 3 & 13235 & -89 & 13146 \\
& $6 d_{5 / 2}$ & 13110 & -5 & 3 & 13108 & -44 & 13064 \\
\hline \hline
\end{tabular}

${ }^{a}$ Brueckner orbitals with the all-order $\hat{\Sigma}$ including ladder diagrams.

${ }^{b} \Delta=E_{\exp }-E_{\text {calc }}$

The results for the Breit and QED corrections are presented in Table IV.

\section{Discussion}

Final results of the calculations of the energy levels of cesium and thallium are presented in Table IV They include the all-order correlations considered in section II. ladder diagrams included into the correlation potential $\hat{\Sigma}$, Breit and QED corrections. The difference between theory and experiment $(\Delta)$ is a fraction of a per cent in all cases. This represents significant improvement comparing to the correlation potential method considered in section II.

It is important that the accuracy is now about the same for all states of both atoms. This proves the claim that poor accuracy for $d$ states of cesium and $p$ states of thallium in the all-order correlation potential method (section III) is due to the poor treatment of the residual Coulomb interaction between external electron and the core. Inclusion of this effect via ladder diagrams leads to significant improvement for these states while it has little effect on the states where accuracy is already high.

In the case of thallium the main source of uncertainty is the choice of screening factors for calculation of the exchange diagrams (Table I). Relative contribution of the exchange correlation diagrams for thallium is larger than for cesium. Therefore, approximate inclusion of higherorder correlations into exchange diagrams via the use of screening factors works very well for cesium but not so well for thallium. One possible cause of action for further improvement is the use of the Feynman diagram technique for the exchange diagrams as well as for direct diagrams as it was done in our calculations of the PNC in cesium [18].

The efficiency of the present method of calculations is the same as for the standard SD approximations. Although the equations (3) and (4) have fewer terms than the SD equation the calculations are strongly dominated by a singe term which has double summation over states above the core. This term exists in both cases, the equations (3) and (4) for ladder diagrams and in the standard $\mathrm{SD}$ equations. Computer resources needed for the calculation of the all-order correlation potential $\hat{\Sigma}$ are practically negligible compared to the iterations of the equations (3) and (4). It would be correct to say that the use of the all-order correlation potential method in combination with the equations similar to the SD equations is a way to include important triple and higher excitations without affecting the efficiency of the calculations. Another way of describing the method is to state that it adds one more class of the higher-order correlations to the all-order correlation potential method. This class represents residual Coulomb interaction of the external electron with atomic core.

\section{CONCLUSION}

The all-order correlation potential method is extended to include one more class of higher-order diagrams to all orders. This class describes residual Coulomb interaction of an external electron with atomic core and represented by ladder diagrams. This is in addition to such higherorder effects as screening of Coulomb interaction between atomic electrons by core electrons, interaction between an electron excited from the core with a hole created by this excitation and the iterations of the correlation operator. Calculations of the energy levels of cesium and thallium show significant improvement in the accuracy. This opens a way of more accurate calculations for many important applications.

\section{Acknowledgments}

The work was supported in part by the Australian Research Council.
[1] J. Guena, M. Lintz, and M. A. Bouchiat, Mod. Phys. Lett. A, 20, 375 (2005).

[2] J. S. M. Ginges and V. V. Flambaum, Physics Reports, 397, 63 (2004).

[3] V. V. Flambaum, Int. J. Mod. Phys. A 22, 4937 (2007).
[4] S. N. Lea, Rep. Prog. Phys., 70, 1473 (2007).

[5] J-P. Uzan, Rev. Mod. Phys. 75, 403 (2003).

[6] J. L. Hall, ChemPhysChem, 7, 2242 (2006).

[7] T. W. Hansch, ChemPhysChem, 7, 1170 (2006).

[8] C. S. Wood et al, Science 275, 1759 (1997). 
[9] P. A. Vetter at al, Phys. Rev. Lett. 74, 2658 (1995).

[10] V. A. Dzuba, V. V. Flambaum, P. G. Silvestrov, O. P. Sushkov, J. Phys. B: At. Mol. Phys., 20, 3297-3311 (1987).

[11] M. G. Kozlov, S. G. Porsev, and W. R. Johnson, Phys. Rev. A 64, 052107 (2001).

[12] V. A. Dzuba, V. V. Flambaum, and M. G. Kozlov, Phys. Rev. A, 54, 3948 (1996).

[13] V. A. Dzuba, V. V. Flambaum, O. P. Sushkov, Phys. Lett A., 140, 493-497 (1989).

[14] V. A. Dzuba, V. V. Flambaum, O. P. Sushkov, Phys. Lett. A, 141, 147-153 (1989).

[15] V. A. Dzuba, V. V. Flambaum, A. Ya. Kraftmakher, O. P. Sushkov, Phys. Lett. A, 142, 373-377 (1989).

[16] V. A. Dzuba, V. V. Flambaum, O. P. Sushkov, Phys. Rev. A, 51, 3454 (1995).

[17] V. A. Dzuba, V. V. Flambaum, J. S. M. Ginges, Phys. Rev. A, 63, 062101 (2001).

[18] V. A. Dzuba, V.V. Flambaum, J.S.M. Ginges, Phys. Rev. D, 66, 076013 (2002).
[19] M. S. Safronova and W. R. Johnson, Adv. At. Mol. Opt. Phys. 55, 191 (2008).

[20] S. A. Blundell, W. R. Johnson, and J. Sapirstein, Phys. Rev. Lett. 65, 1411 (1990); S. A. Blundell, J. Sapirstein, and W. R. Johnson, Phys. Rev. D 45, 1602 (1992).

[21] A. Derevianko, and S. G. Porsev, Europ. Phys. J. A, 32 517 (2007).

[22] R. Pal, M. S. Safronova, W. R. Johnson, A. Derevianko, and S. G. Porsev, Phys. Rev. A 75, 042515 (2007).

[23] C. E. Moore, Atomic Energy Levels Natl. Bur. Stand. (US), Circ. No. 467 (U.S. GPO, Washington, DC, 1958), Vol. III.

[24] S. A. Blundell, W. R. Johnson, Z. W. Liu, and J. Sapirstein, Phys. Rev. A 40, 2233 (1989).

[25] V. A. Dzuba, V. V. Flambaum, P. G. Silvestrov, O. P. Sushkov, J. Phys. B: At. Mol. Phys., 20, 1399-1412 (1987).

[26] V. V. Flambaum and J. S. M. Ginges, Phys. Rev. A 72, 052115 (2005). 